\title{
Extensión crítica e in tegralidad de funciones en las prácticas de enseñanza de portugués como lengua de integración regional en una escuela de gestión social
}

Critical extention and functional integration of teaching practices of Portuguese as a regional integration language in a social management school

Natalia Ricciardi ${ }^{1}$

Virginia Rubio Scola ${ }^{2}$

Dalila Capeletti ${ }^{3}$

Thalita Camargo Angelucci ${ }^{4}$

Pablo García Giménez ${ }^{5}$

\section{Resumen}

El presente artículo busca recuperar y analizar, mediante una sistematización de experiencias, aprendizajes surgidos en una práctica extensionista de la Escuela de Lenguas de la Universidad Nacional de Rosario (U.N.R.) con actores de una escuela de gestión social de la periferia de Rosario. Esta práctica se concretó por medio del proyecto de Compromiso Social Universitario "Portugués e integración" que procuró acercar a los estudiantes de esta escuela al portugués como lengua de integración, buscando favorecer las reflexiones sobre la construcción de una ciudadanía regional. A su vez, procuramos fomentar en los estudiantes universitarios la reflexión crítica y

Recibido: 30 de abril de 2020 Aceptado: 4 de julio de 2020 Publicado: 10 de julio de 2020

${ }^{1}$ Profesora de Portugués. Actualmente titular de las cátedras de Metodología de Enseñanza de la Lengua Extranjera y Lengua y Gramática Portuguesa III de la Escuela de Lenguas de la Universidad Nacional de Rosario, Argentina. Doctoranda en Lingüística y Lenguas (FHUMYAR-UNR). Correo electrónico: natalia.ricciardi@gmail.com

${ }^{2}$ Magíster en Lingüística. Profesora Adjunta de Lingüística General e Introducción a la metodología y a las técnicas de la investigación de la Licenciatura en portugués en la Facultad de Humanidades y Artes, Universidad Nacional de Rosario, Argentina, doctoranda de Lingüística en la Universidad de Buenos Aires. Correo electrónico: virginrubio@gmail.com

${ }^{3}$ Licenciada en Relaciones Internacionales. Universidad Nacional de Rosario. Argentina. Correo electrónico: dalilacapeletti75@hotmail.com

${ }^{4}$ Psicóloga (UFSCar-Brasil), Doctoranda en Ciencias de la Educación (FHUMYAR-UNR) y JTP en la cátedra Trabajo de Campo en la misma institución. Facultad de Humanidades y Artes (UNR) e Instituto Rosarino de Investigaciones en Ciencias de la Educación (IRICE). Rosario, Argentina. Correo electrónico: angelucci@iriceconicet.gov.ar

${ }^{5}$ Ingeniero agrónomo, especialista en Gobernabilidad y Derechos Humanos. Actualmente Director de formación y capacitación del Área de Extensión y Territorio de la Universidad Nacional de Rosario. Correo electrónico: pgarciagimenez@gmail.com 
la acción transformadora a través de la implementación de un programa que contemplase la realidad social, cultural y económica de la comunidad. Para ello, nos posicionamos en la línea teórica de la Extensión Crítica, entendiendo que la integralidad de funciones (docencia, extensión e investigación) es fundamental para promover un proceso formativo integral.

Palabras clave: extensión crítica, portugués en la escuela media, integración regional, sistematización, integralidad de funciones

\section{Abstract}

This article seeks to reflect upon and analyze, through an incipient systematization of experiences, the learning experience of students from a social management school on the outskirts of Rosario. The project of CSU "Portuguese and integration" of the Languages Program of the UNR, seeks to promote reflection on the construction of regional citizenship by presenting Portuguese as a "language of integration". We also try to encourage critical thinking and transformative action within university students through the implementation of a program that evokes contemplation about the community's social, cultural and economic reality. In order to achieve our goals, we assume the theoretical assumptions of "Critical Extension", and we firmly believe that the integration of functions (teaching, extension and research) should be always considered as an integral training process.

Keywords: Critical Extension, Portuguese language in middle school, Regional integration, Systematization, Integrality of functions

\section{Introducción}

El vínculo entre universidad y sociedad, conocido como "Extensión Universitaria”, convoca a la universidad a comprometerse con la transformación social, aproximando la producción de saberes a la sociedad con el objetivo de superar la asimetría en la apropiación del conocimiento, ciencia y tecnología (De Paula, 2013). Si bien el surgimiento de la extensión universitaria como tal remonta a mediados del siglo XIX en Inglaterra, actualmente se ha transformado y viene ganando espacio en los países de nuestra región.

En Latinoamérica, la problematización sobre la extensión se inició con la lucha por la Reforma Universitaria impulsada en el año 1918 en Argentina, como efecto de la Revolución Mejicana de 1910. Las universidades inauguradas hasta ese entonces se caracterizaban por su orientación elitista, dando la espalda a los problemas sociales, económicos, políticos y culturales de su contexto. A partir de 1960 se agudizó la lucha 
de clases por influencia de la Revolución Cubana, reivindicando tanto la identidad nacional como la socialización de los medios de producción. En respuesta, emergieron en diversos países latinoamericanos gobiernos dictatoriales que gobernaron hasta fines del siglo XX. Influenciada por las reivindicaciones sociales y en reacción a los gobiernos autoritarios surge la extensión universitaria en su vertiente crítica (Freire, 1987; Fals Borda, 1986).

Resulta importante marcar las diferencias conceptuales que existen entre las tradicionales prácticas de voluntariado (que tienen como objetivo explícito desarrollar tareas de "solidaridad" con sectores vulnerables) de las experiencias de extensión universitarias, en donde se busca no solo aportar a la construcción de soluciones a problemáticas comunitarias, sino también lograr una coproducción de conocimientos en un nuevo acto educativo, con nuevas prácticas en torno a la enseñanza. Este camino en doble sentido es condición sine qua non de las prácticas extensionistas, mientras que las de voluntariado pueden sostenerse, simplemente y sin contradicciones, en el altruismo y la filantropía.

Las prácticas llevadas adelante con este proyecto se enmarcan en la línea teórica de la Extensión Crítica, definida como "un proceso educativo transformador donde no hay roles estereotipados de educador y educando, donde todos pueden aprender y enseñar" (CDC, 2009, s/p). En su dimensión pedagógica, constituye una metodología de aprendizaje integral y humanizadora. La universidad, mediante esta función sustantiva, reconoce situaciones que generan en sus propios actores procesos de sensibilización, humanización y solidaridad, pero que también impulsan resultados académicos orientando líneas de investigación y planes de estudio, gestando compromiso universitario con la sociedad y con la resolución de sus problemas.

Asumimos esta vertiente de la extensión, incorporando la noción de "diálogo de saberes" (Sousa Santos, 2009; Castro-Gómez, 2007). En esta dirección, realizamos la sistematización del proyecto de extensión "Portugués e integración" 6 perteneciente a la Convocatoria 2017 “Compromiso Social Universitario” de la Secretaría de Políticas Universitarias del Ministerio de Educación de la Nación. Enmarcado en las carreras de portugués (Profesorado, Licenciatura y Traductorado) de la Escuela de Lenguas de la Universidad Nacional de Rosario, el proyecto se conformó con estudiantes y profesores de diferentes disciplinas y carreras además de las mencionadas (relaciones internacionales, ciencias de la educación, estudios culturales, agronomía y letras). El

\footnotetext{
${ }^{6}$ Agradecemos especialmente la participación de Ma Cecilia Postiglione; Joana Arman; Cíntia Chagas,de Cássia; Débora Valenti; Ana Lucia Ysasi; Micaela de Figueiredo Peralta; Estefanía de la Rosa; Leticia Bergero; Luisina De María; Nicolle Freire; Bárbara Montaña; Lourdes Martínez; Jésica Rodríguez, Melina Fussi; Aldana Katsicaris; Rita Achinelli; Luciana Demkura; Yoana Sandoval, y Valeria Cuadrado, quienes con su apoyo hicieron posible el desarrollo de este proyecto extensionista. Además, queremos agradecer a los coordinadores y docentes de la escuela ÉTICA con quienes desarrollamos la experiencia.
} 
mismo tuvo como objetivo el acercamiento de los estudiantes de la escuela de gestión social Ética (Escuela de Territorio Insurgente Camino Andado) al portugués como lengua de integración regional fomentando la reflexión sobre el vínculo entre lengua y ciudadanía latinoamericana. A su vez, también buscó fomentar en los estudiantes universitarios la reflexión crítica y la acción transformadora contemplando la realidad social, cultural y económica de la comunidad. A partir de esta experiencia, los y las estudiantes, docentes y profesionales conformamos el grupo de estudios denominado "Lenguas e Integración", que con el objetivo de promover la integración de las tres funciones universitarias -docencia, investigación y extensión-, amplió su campo de acción para incluir en su abordaje otras lenguas de la región, además del portugués (variedades latinoamericanas del español, lenguas originarias y lenguas de inmigración).

En el presente trabajo definimos, en un primer momento, a partir de la experiencia vivida, el objeto, el objetivo y el eje del proyecto, además de presentar el plan de sistematización de las experiencias de acuerdo con el modelo de Jara (2013). A continuación, definimos históricamente el contexto local y regional, para luego describir los momentos más significativos de la experiencia. En un tercer momento, abordamos la integralidad de funciones sustantivas de la universidad a fin de analizar críticamente la experiencia. En un cuarto y último momento, presentamos nuestras reflexiones sobre la experiencia, su impacto en nuestra transformación (autoconocimiento) y futuras líneas de investigación, docencia y extensión.

\section{Sistematización de la experiencia: objeto, objetivo, eje y plan}

Al preguntarnos por qué sistematizar la experiencia, retomamos las consideraciones de Jara (2013), quien afirma que existe cierta confusión a la hora de entender qué significa la sistematización. En algunas disciplinas se refiere, principalmente, a ordenar datos e informaciones. Desde la educación popular y los proyectos sociales se utiliza el mismo término aplicado también a experiencias; por eso, no hablamos de "sistematización" a secas, sino de "sistematización de experiencias". Esto implica un esfuerzo de comprensión profunda de las prácticas realizadas para que la tarea reflexiva permita recuperar organizadamente la praxis, realizar ajustes $y$ modificaciones, teorizar sobre la práctica y, además, comunicar el conocimiento producido (Ghiso, 1998). Si bien se reconoce que dicho proceso es complejo y de larga duración, una aproximación al mismo fue la escritura del presente trabajo para repensar las próximas experiencias. De acuerdo con el esquema de la Metodología de la Sistematización (Zúñiga Lopez y Zúñiga Preciado, 2013) presentado en la Figura 1, definimos nuestro objeto, esto es, la experiencia de extensión Portugués e 
Integración llevada a cabo desde octubre del 2017 a octubre del 2019, en la ciudad de Rosario, con la Escuela de Gestión Social, La ÉTICA. En este sentido, el objetivo de esta sistematización fue fortalecer la práctica extensionista en las carreras de portugués a fin de promover la investigación situada y una docencia comprometida con su contexto. Definimos, en consecuencia, nuestro eje sobre la articulación de las funciones de la universidad (docencia, investigación y extensión) a partir de la práctica extensionista asumiendo, desde la extensión crítica, la integralidad como política universitaria.

Figura 1: Síntesis Metodológica del Proceso de Sistematización

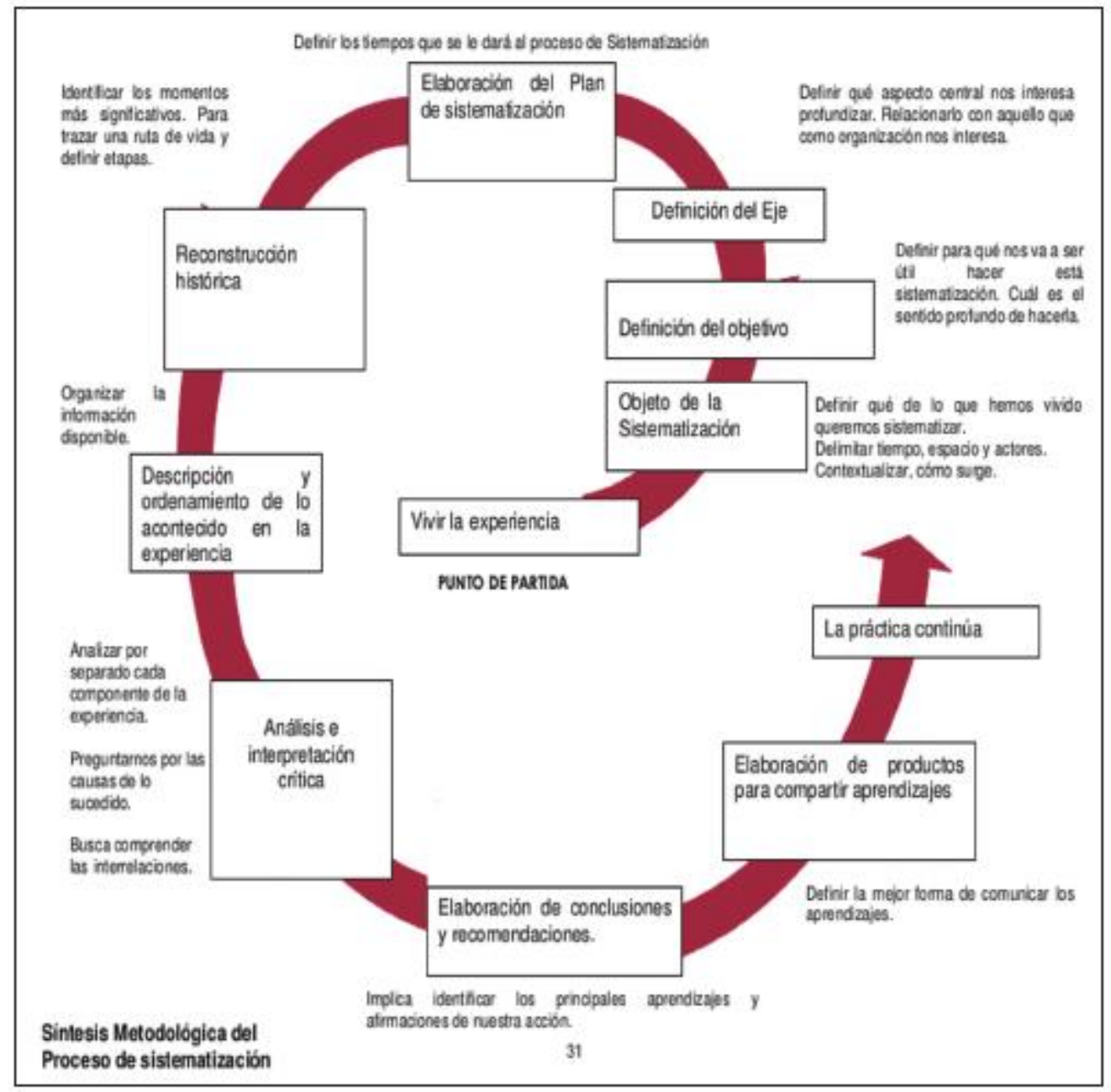

Fuente: Zúñiga Lopez y Zúñiga Preciado, 2013, p. 31

El proceso de sistematización fue acompañando la experiencia a partir de la reflexión permanente sobre la práctica extensionista. Esta reflexión resultó en la elaboración de diferentes productos que nos permitieron compartir los aprendizajes a partir de la socialización en diferentes espacios colectivos. Una vez concluído el proyecto de voluntariado universitario (octubre del 2019), se conformó un grupo de estudios e investigación en el que nos propusimos profundizar la formación en Extensión 
Crítica y en el cual surgió el debate en torno a las problemáticas de la integralidad de las funciones en la universidad. A su vez, en el marco del aislamiento social obligatorio establecido a partir de marzo del 2020 en Argentina, llevamos adelante reuniones virtuales periódicas y ampliamos las lecturas teóricas que se venían realizando, lo que nos motivó a realizar una sistematización de la experiencia extensionista materializada en el presente artículo.

\section{El portugués y la integración regional: la experiencia de enseñanza de portugués en la ÉTICA}

La conformación de integraciones regionales entre los países latinoamericanos Mercosur (Mercado Común del Sur, 1991), Unasur (Unión de Naciones Suramericanas, 2008), Celac (Comunidad de Estados Latinoamericanos y Caribeños, 2010)- con proyectos políticos que buscaron ir más allá de los acuerdos comerciales, se reflejó en políticas educativas nacionales. En Argentina, la Ley de Educación Nacional (2006) contempló al Mercosur como contenido obligatorio y asumió una perspectiva latinoamericana para la enseñanza formal. Asociadas a estas iniciativas, se sancionaron las leyes de oferta obligatoria de español en la escuela secundaria en Brasil (Ley n. ${ }^{\circ}$ 11.161/2005-2017) y de portugués en la escuela secundaria en Argentina (Ley n. ${ }^{\circ}$ 26.468/2009). Sin embargo, la ley de portugués en nuestro país, con plazo de implementación hasta 2016, no se cumplió. El estatuto de obligatoriedad no fue contemplado en los Núcleos de Aprendizajes Prioritarios de Lenguas Extranjeras del Ministerio de Educación de la Nación y, actualmente, escasas escuelas presentan la opción del portugués como lengua extranjera (Rubio Scola, 2019a).

Además, como fue analizado en Rubio Scola (2019b), los materiales didácticos que circularon en las pocas escuelas que implementaron el portugués han vehiculado representaciones sociales que no priorizaron una visión de la lengua portuguesa como lengua de la integración regional, incluso en la etapa de mayor voluntad política de acercamiento entre los gobiernos de América Latina (2003-2015). Por un lado, en la mayoría de los manuales de portugués, se suele privilegiar representaciones que asocian el portugués con una lengua de comercio o de turismo. De esta forma, se concibe un destinatario del manual como un sujeto abstracto, no necesariamente argentino o hispanohablante, que busca aprender portugués de Brasil para conocer su cultura en términos folklóricos y patrimoniales con una finalidad instrumental y mercantil. Asimismo, los manuales asocian la lengua de Brasil con un exacerbado nacionalismo brasileño, que en lugar de funcionar como vínculo con los países vecinos, termina operando como frontera, ya que se concibe a Brasil de forma aislada de la región (Rubio Scola, 2019b). Por otro lado, otros materiales didácticos, si bien cuestionan esta concepción y proponen otra noción de lengua indisociable de sus 
hablantes y sus culturas, priorizan una ideología más global que visibiliza el área idiomática, la lusofonía, en la que el estatus regional del portugués queda borrado. Creemos que esto refleja la falta de concientización sobre el proyecto regional y sobre el papel central que las lenguas pueden ocupar en la construcción de una identidad latinoamericana. Por lo tanto, sostenemos que es necesario sensibilizar sobre la enseñanza de las lenguas mayoritarias de la región (portugués y español) en sus variedades regionales. Defendemos que esta concientización también repercutiría en la valoración de las lenguas originarias y de inmigración, siendo muchas de estas compartidas por diferentes países de la región.

Ante este panorama y preocupados por la disolución de los organismos políticos de cooperación regional, como fue el caso de Unasur, y por la alineación ciega de Brasil con Estados Unidos, el proyecto que llevamos adelante se propone visibilizar la enseñanza del portugués en tanto lengua de integración regional en la escuela secundaria. Consideramos que es importante que haya, a nivel macro político, una legislación lingüística que responda a la dimensión identitaria del proyecto regional; pero, además, sostenemos que para que esta pueda implementarse, se hacen necesarias acciones a nivel micropolítico que intervengan en las representaciones sociales del portugués en tanto lengua a ser enseñada en las escuelas de nivel medio. Por esta razón, hemos proyectado una prueba piloto en una escuela de gestión social de nivel medio. Esta escuela está localizada en el barrio Nuevo Alberdi en la periferia de la ciudad de Rosario. Asisten alrededor de cien estudiantes jóvenes adultos de las inmediaciones de la escuela. Las clases son dictadas por profesionales, profesores voluntarios ad honorem comprometidos con el el proyecto de transformación social del barrio. La escuela funciona desde el año 2011, pero recién en junio del 2018, año en el que realizamos la experiencia, se concretó su oficialización como la primera escuela con características de gestión social de la provincia de Santa Fe registrada como Eempa particular $N^{\circ} 3.188$. Todas las decisiones de la escuela se realizan por asambleas, en las que se define una currícula con materias y seminarios, cuyos contenidos se dictan de forma interdisciplinaria y por intermedio de parejas pedagógicas. El seminario propuesto, "Portugués y ritmos musicales", se dictó entre una profesora de la institución y dos voluntario/as estudiantes de la carrera de Profesorado en Portugués de la Escuela de Lenguas de la Facultad de Humanidades y Artes - Universidad Nacional de Rosario (UNR).

La experiencia consistió en el dictado del seminario de portugués, asumiendo su estatus de lengua de integración regional con el fin de cuestionar lugares comunes y estereotipos relacionados con ella. El proyecto surgió en el marco de las carreras de portugués de la UNR (además del profesorado, la Escuela de Lenguas cuenta con el traductorado y la licenciatura en portugués) y se desarrolló sobre tres ejes 
orientadores: fomentar la investigación interdisciplinaria y la formación permanente de sus miembros (profesores y estudiantes); incentivar la participación en las actividades extensionistas, tanto de voluntarios como de profesores de la Universidad, en diálogo permanente con los profesores y estudiantes de la escuela de gestión social; y proponer, a partir de la experiencia en territorio, líneas de pensamiento y trabajo para la elaboración de material didáctico volcado a la enseñanza de portugués, que contemplen la realidad local y refuercen las representaciones de la lengua portuguesa en tanto lengua vecina y lengua de integración.

La planificación de las actividades a desarrollarse en el territorio fueron, desde un principio, planteadas y discutidas en reuniones conjuntas con los coordinadores y profesores de la escuela de gestión social, y este diálogo permanente nos permitió coconstruir la propuesta y replantearla permanentemente a partir de los aprendizajes que pudimos adquirir en la práctica permanente de escucha, reflexión, evaluación y reformulación.

Antes de iniciar la experiencia en territorio, propusimos varias reuniones de lectura y discusión que permitieron entender más sobre la realidad regional, los acuerdos multilaterales, la historia del Mercosur, la legislación lingüística, las políticas editoriales y el currículo escolar en la región, el país y la provincia. Las reuniones se dieron de forma participativa y dieron lugar a permanentes planteos y nuevas ideas. Con el correr de la experiencia, los encuentros fueron asumiendo otras formas, como talleres de capacitación, jornadas ${ }^{7}$ de reflexión y de formación, que se abrieron a toda la comunidad educativa de la universidad.

Buscamos, también, fomentar el diálogo y las actividades participativas entre los voluntarios y los estudiantes de la escuela, para lo cual se programaron visitas al barrio y a la casa donde funciona el proyecto educativo de la ÉTICA. Estas actividades apuntaban a la sensibilización sobre las diferentes realidades sociales que allí se presentaban. También se programó una visita de los estudiantes de la ÉTICA a la Facultad de Humanidades y Artes, que tuvo como objetivo acercarlos a la realidad universitaria y presentarles nuestro espacio de estudio y trabajo ${ }^{8}$.

En uno de los encuentros, los voluntarios del proyecto de extensión invitaron a los estudiantes con algunos dulces típicos brasileños que ellos mismos prepararon, para acompañar la presentación de un cortometraje en portugués, Cidade dos Homens, que muestra un baile funk en la favela carioca. A partir de la visualización del corto, los

\footnotetext{
${ }^{7}$ Nos referimos a la organización de las siguientes actividades: Jornada-taller de Actualización en Extensión universitaria y Socialización del Proyecto Portugués e Integración; Jornada de Formación sobre Ciudadanía Regional y Análisis de Materiales Didácticos para la Enseñanza de Portugués.

${ }^{8}$ La ÉTICA va a la facultad. Proyección y debate del corto: Cidade dos homens: Sábado (Dir: Fernando Meirelles, año 2003).
} 
estudiantes pudieron reconocer(se) en muchas situaciones, problematizar la violencia de género, las relaciones de amor y amistad, y compartir sus impresiones y experiencias con los voluntarios. El interés provocado por el funk nos llevó a replantear qué géneros musicales trabajar en el taller, que en principio se habían pensado en torno a los que considerábamos más representativos de la MPB (Música Popular Brasileña), como el Samba o la Bossa Nova, y, en cambio, elegimos el Funk, Rap, Forró y Sertanejo, géneros asociados a zonas periféricas.

El dictado del taller de Portugués y Ritmos Musicales se realizó todos los lunes de los meses de abril, mayo y junio del año 2018. Al finalizar, realizamos la celebración de la Fiesta de San Juan o Festa Junina, que se concibió de forma participativa e intercultural con características brasileñas y argentinas.

Ante la sugerencia de los coordinadores y profesores de la escuela ÉTICA de extender nuestra experiencia al nivel inicial, las prácticas extensionistas continuaron durante los meses de agosto y septiembre en el Jardín de Infantes. Si bien esta última participación escapaba a nuestro objetivo inicial de llevar el portugués a los espacios de la educación media, la experiencia de sensibilización hacia una lengua diferente con niños y niñas que, en muchos casos provenían de zonas rurales cercanas a la ciudad de Rosario, fue motivadora. A través de juegos, disfraces, canciones y relatos de cuentos infantiles en portugués, se desarrolló una incipiente experiencia que propuso nuevos e impensados desafíos.

Las actividades del proyecto también se expandieron a la participación en congresos y eventos académicos. La exposición en la mesa coordinada Debatiendo la integración regional en América Latina: ¿es posible pensar una ciudadanía regional?? nos permitió socializar el proyecto, debatir con colegas de otras carreras y abrir nuevas propuestas de intervención e investigación. Además, participamos en las IV Jornadas Descobrindo Culturas em Língua Portuguesa, en la Universidad Nacional de Córdoba (noviembre, 2018). En el año 2019 se presentó la experiencia en el X Coloquio PELSE (Portugués y Español como Lengua Segunda y Extranjera) de AUGM (Asociación de Universidades Grupo Montevideo) a través de una ponencia y una sesión de posters que fueron elaborados y presentados por los voluntarios. El encuentro contó con docentes e investigadores de Brasil, Uruguay y Argentina, y los voluntarios tuvieron la oportunidad de compartir sus experiencias con profesores y alumnos de diferentes universidades de la región.

En relación al tercero de nuestros ejes -la reflexión sobre el material didáctico disponible en la región para la enseñanza del portugués con el objetivo de repensar las propuestas para la enseñanza del idioma en tanto lengua de la integración

\footnotetext{
${ }^{9}$ En el contexto del Congreso Nacional e Internacional sobre la Democracia, organizado por la Facultad de Ciencias Políticas y RRII de la UNR, septiembre 2018.
} 
regional-, trabajamos sobre textos auténticos pertenecientes a diferentes géneros textuales. Entendemos que "la finalidad general de la enseñanza de lenguas apunta al dominio de los géneros, en tanto instrumentos de adaptación y participación en la vida social/comunicativa, y a los aprendizajes relativos a la sintaxis o al léxico como apoyo técnico para esa finalidad global” (Bronckart \& Dolz, 2007, p.158). Es en esa dirección que planteamos el abordaje de textos empíricos en lengua portuguesa que forman parte de la cultura y realidad social vivida por los alumnos (canciones de rap, videoclips musicales, historietas y grafittis). Este abordaje se apoya en los conocimientos de los alumnos sobre géneros textuales en la lengua materna y en otras lenguas extranjeras y permite una reflexión intercultural, que problematiza nociones de ciudadanía, inclusión, respeto y conocimiento del otro.

\section{Integralidad de las funciones universitarias: análisis crítico de la experiencia extensionista}

En cuanto a la integralidad de las funciones, además de la docencia, la extensión y la investigación deberían ser parte de la metodología de enseñanza universitaria, lo que haría que el proceso formativo fuese integral, a través del contacto directo con la realidad social (UDELAR, 2010) ${ }^{10}$. El punto de partida de ese diálogo, según Freire (1987), consiste en la selección de temas generadores que tengan un potencial contenido programático, es decir, que puedan funcionar como disparadores y generar su propia agenda de actividades. Se busca conformar un programa de trabajo que tenga como horizonte una perspectiva de liberación, conociendo la percepción de la realidad de los sujetos que participan. En nuestro caso, el equipo que elaboró el material didáctico inicial (en base a las anticipaciones sobre las representaciones que los estudiantes de la ÉTICA tenían sobre Brasil) pudo conjugar esta selección con los conceptos de "música" y "cultura", a partir de los cuales pudieron abordarse contenidos gramaticales (como presentación personal y uso de pronombres personales), y también cuestiones de otro orden, como identidades individuales, identidades colectivas, el auto-reconocimiento, problemas de violencia institucional, violencia de género, entre otros.

El desafío de la institucionalización de la extensión como parte de la cotidianeidad universitaria se enlaza con uno de mayor envergadura, que es el de lograr las condiciones de posibilidad para que en todas las disciplinas, la integralidad forme parte de la actividad docente y esto impacte en la transformación de los procesos de formación y de producción de conocimientos de los estudiantes. Ambos desafíos

\footnotetext{
${ }^{10}$ En el caso de la Universidad Nacional de Rosario, la extensión está reconocida como un pilar fundamental en su estatuto, sin embargo, de forma general, en la práctica docente no está incorporada como una estrategia de educación.
} 
implican partir de una concepción diferente, no aislada, generalizada en toda la universidad y en diálogo con las otras funciones universitarias. Se puede aprender fuera de las cuatro paredes de un salón; se puede aprender con otros; se puede aprender y enseñar de forma diferente, y necesariamente se aspira a trabajar por mejores condiciones de vida para todos los ciudadanos y las ciudadanas desde la Universidad Pública.

La práctica en el territorio nos permitió retroalimentar nuestra labor docente como así también profundizar nuestras investigaciones. La experiencia indica que cuando la extensión se realiza integrada a las demás funciones y partiendo de concepciones como las mencionadas anteriormente -en la práctica cotidiana y concreta, con estudiantes en terreno, con actores sociales concretos- se está modificando el acto educativo en el cual se está inmerso. En la actualidad, la mayor parte del tiempo de aprendizaje del ámbito universitario está articulada en torno a espacios de docencia. Los estudiantes pueden atravesar algún espacio de investigación ya sea por interés personal (en un número muy reducido), o bien periféricamente, bajo la forma de materias integradoras finales, tesinas o similares, en una instancia de la vida universitaria donde todas las demás disciplinas se han desarrollado de la manera tradicional. Con la extensión universitaria, la situación es incluso más limitada, ya que ocupa espacios marginales dentro de la organización de los tiempos académicos y planes de estudio (si los ocupan), o está impulsada por paradigmas iluministas y/o transferencistas que se contraponen a lo propuesto desde la Extensión Crítica.

En este sentido, para evitar caer en sectarismos fundamentalistas, cualquier avance en propuestas coherentes con las líneas teóricas mencionadas es valorado muy positivamente. El proyecto aquí presentado surgió como resultado de procesos de investigación de las docentes coordinadoras provenientes de las carreras de portugués, con base realmente interdisciplinaria y participación voluntaria de estudiantes de distintas disciplinas de las carreras de la Universidad Nacional de Rosario. También resultó un desafío para el equipo no permanecer en el lugar cómodo que la enseñanza "tradicional" del portugués como lengua extranjera representa bajo el imaginario colectivo que describimos anteriormente, con ideas estereotipadas y concepciones de lengua reduccionistas, sobre todo en su relación con la dimensión cultural. Al mismo tiempo, la experiencia implicó el aprendizaje y discusión sobre políticas lingüísticas y metodologías de enseñanza, buscando ir más allá de las competencias meramente instrumentales y gramaticales.

En este sentido, podemos presentar los aprendizajes conseguidos a partir de la práctica en función del área de trabajo específica de cada integrante del proyecto. En general, interpelamos el rol docente en su formato tradicional y unidireccional, que lo plantean como el detentor del saber que acerca los conocimientos planificados y 
programados a los alumnos. La experiencia nos llevó a reconsiderar nuestras prácticas a partir de la reflexión permanente, de la consideración de las necesidades e intereses que se planteaban en el desarrollo de cada clase, y de la concientización del impacto que las características psicológicas y sociales de los alumnos tenían en la forma de plantear los temas, los textos y la metodología de enseñanza de la lengua (incluyendo técnicas y actividades). Asimismo, la experiencia extensionista se reflejó en la propia actividad docente de las materias de la currícula de las carreras de portugués, especialmente aquellas relacionadas a la formación docente, en la medida que el intercambio de saberes mediados por la práctica docente vivenciada en territorio interpeló los contenidos curriculares. Esto fomentó la permanente reflexión crítica sobre la propia práctica, el cuestionamiento de técnicas de enseñanza y actividades de aprendizaje "comunicativas", y la revisión de conceptos tales como currículum, planificación, objetivos de aprendizaje, lenguas, variedades sociolingüísticas, representaciones sociales respecto a las lenguas, conocimientos y saberes previos e interculturalidad.

Siguiendo estas líneas de reflexión, las estrategias diseñadas para alcanzar los objetivos consensuados con los miembros de la escuela ÉTICA fueron pensadas y revisadas permanentemente, y, por tal motivo, modificadas en más de una ocasión. Entender que para los alumnos de la escuela resultaba más significativo un género musical como el rap, obligó a modificar la forma y los contenidos que inicialmente se pensaron para trabajar, pero también dispararon interrogantes académicos sobre las formas de producción de estos discursos, su problematización como géneros textuales, las herramientas lingüísticas usadas, el estudio comparativo de los mismos en español y en portugués, entre tantos otros temas que pueden transformarse en futuras líneas de investigación.

Las estrategias escogidas también tuvieron que modificarse en el transcurso del desarrollo de las actividades, ya que por pedido de la escuela ÉTICA se pasó de trabajar con estudiantes adolescentes y/o adultos jóvenes a pensar actividades en portugués para los niños del jardín de infantes. Esto se corresponde con procesos de construcción de la demanda junto a la organización social co-participante; pero, a su vez, dificultó la concreción de los objetivos referentes a la construcción de la identidad e integración latinoamericana, ya que estos habían sido pensados para la secundaria, etapa educativa en la que tradicionalmente se busca formar al/ a la ciudadano/a.

Sumado a esto, existieron importantes reflexiones en la producción teórica del proyecto. El diálogo interdisciplinar ha sido nutritivo y la práctica nos ha demandado una construcción de conocimiento en contexto. Una de las primeras líneas de investigación que se abrió fue en torno a la perspectiva crítica de la extensión. Como mencionamos a lo largo de este artículo, fue fundamental para nosotros conocer los 
trabajos que se vienen realizando en torno a la Extensión Crítica y participar de talleres y espacios de formación. Esto resultó esencial a la hora de reflexionar sobre nuestras propias prácticas en el momento de desarrollar el proyecto y en gran parte este acercamiento nos alentó, ya que ayudó a problematizar todas aquellas cuestiones que veníamos experimentando a partir de este diálogo entre la teoría y la práctica. Además de eso, nos orientó con respecto a dificultades metodológicas que se nos presentaban.

Otra línea de estudio en la que este diálogo intercultural nos llevó a incursionar fue la perspectiva decolonial. En este punto, hemos recuperado aportes conceptuales de autores como Boaventura de Sousa Santos (2009), Anibal Quijano (2013, 2000, 1992), Edgardo Lander (2000), Catherine Walsh (2008) y Castro-Gómez (2007). El encuentro con estas teorías y categorías de análisis ha sido novedoso, dado que accedimos a conceptos que responden a preguntas que nos veníamos haciendo cada quien desde su disciplina. La colonialidad del poder en su vertiente epistemológica, es decir, la colonialidad del saber, ha abonado un campo de investigación interdisciplinar que estamos transitando. Pensar formas "otras" de construir conocimiento es una tarea que nos encuentra forjando colectivamente. Y esto es lo que nos invita a continuar en la práctica extensionista, entendiendo que sólo el diálogo de la teoría con la práctica -y viceversa- habilita la producción de un conocimiento genuinamente nuevo, que no sea la reproducción de un saber hegemónico que extiende las desigualdades sociales, sino un corpus teórico práctico que visibiliza y problematiza esas jerarquías partiendo de la realidad concreta.

Por último, se profundizó en la línea de investigación sobre las políticas lingüísticas regionales, con el objetivo de agudizar la mirada crítica y contextualizada sobre los materiales didácticos existentes y los materiales didácticos elaborados por nosotros mismos para el dictado del taller. Este proceso fue guiado a partir de lecturas sobre el análisis del discurso y la perspectiva glotopolítica (Arnoux, 2017). Esta última permite pensar las acciones sobre el lenguaje considerándolas como fenómenos ideológicosdiscursivos (Del Valle, 2007), lo que significa abordarlas en relación dialógica con el contexto sociohistórico. Como vimos, los discursos sobre la lengua portuguesa pueden responder a diferentes intereses políticos, ya que a través de estos se pueden reforzar diversos tipos de integraciones regionales, como son: por un lado, una enseñanza meramente instrumental que privilegiaría un vínculo comercial y turístico, proyectando un destinatario abstracto, posible turista $u$ hombre de negocios; o, por otro lado, una enseñanza que apunte a la construcción de una identidad compartida por los países latinoamericanos y destinada a un ciudadano que, a través del portugués, pueda informarse en medios de comunicación brasileños, 
buscar bibliografía en esta lengua, participar de eventos regionales, etc. (Canteros, 2011).

De esta manera, como muestra la Figura 2, a diferencia de lo que sucede en el acto educativo tradicional, hemos buscado integrar y retroalimentar las tres funciones universitarias.

Figura 2: Acto educativo tradicional (a la izquierda) y con integralidad de funciones (a la derecha)
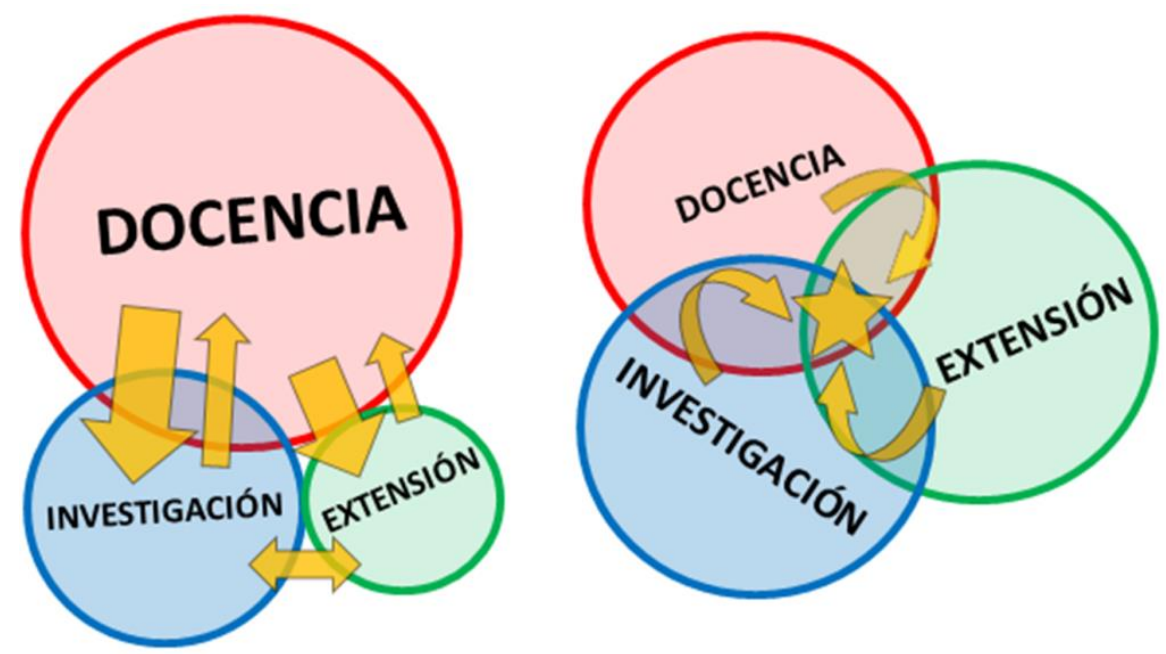

Fuente: elaboración propia

\section{Consideraciones finales}

El proyecto de extensión "Portugués e integración" representó un gran desafío y posibilitó múltiples aprendizajes a los/las universitarios/as que formamos parte de esta experiencia extensionista que, a su vez, se siguen encarnando de forma integrada en nuestro quehacer situado. Para presentar nuestras consideraciones finales, decidimos disgregar estos aprendizajes en tres dimensiones: formativa, subjetiva y proyectiva hacia nuevos diálogos de saberes.

Desde la primera dimensión, la experiencia nos llevó a repensar los procesos de formación del docente de portugués como lengua extranjera. Como es de común conocimiento, la enseñanza de lenguas - maternas y extranjeras- invoca con mucha fuerza el imaginario del dominio gramatical. A pesar de que en nuestra formación asumimos una mirada sociolingüística, el abordaje del portugués como lengua de integración, o lengua de vecindad, representó un gran desafío. Trabajar cuestiones identitarias, tanto personales como colectivas, era uno de los objetivos originales del proyecto así como también la desmitificación de ciertas representaciones. Brasil puede verse más allá de la idea de destino turístico veraniego de la clase media-alta y, 
en igual sentido, el portugués puede entenderse estratégicamente como lengua de integración regional, y no sólo como lengua de turismo. Esta visión se nutrió en la participación interdisciplinaria con los diálogos entre los participantes de distintas carreras de la UNR -letras, relaciones internacionales, agronomía, psicología y ciencias de la educación.

En la segunda dimensión, la experiencia en el territorio impactó en nuestras formas de subjetivación y significó, concretamente, pisar un lugar distinto al nuestro y, luego, mirar y actuar sobre el mundo desde la nueva perspectiva que se habilita. Desde la extensión crítica reafirmamos que la lectura y discusión no son suficientes para una propuesta transformadora. Pisar el territorio es lo que permite vivenciar nuevas prácticas y pararse ante lo desconocido. Desde allí, brotan los intercambios más genuinos y nuevos interrogantes. En este aspecto, muchos universitarios, aún viviendo en la ciudad de Rosario, no conocían el barrio ni la existencia de esta escuela. Las especificidades del territorio despertaron muchas inquietudes que se fueron transformando en diferentes gestos de interpretación sobre el otro y sobre nosotros mismos. Aprender y enseñar se ha configurado desde una dinámica impensada. Impensada, por supuesto, porque no está en el pensar, ni tampoco en el quehacer; está, definitivamente, en el sentipensar. La experiencia se hace cuerpo en la medida que se expone a lo desconocido, lo indisciplinado, lo indómito.

Con respecto a la última dimensión, como fruto de este proceso grupal recogemos un gran lazo que sigue tejiéndose para proyectar otros mundos posibles: el grupo de estudios Lenguas e Integración. Lejos de ser una tarea terminada, otras experiencias permitirán mejorar aún más el acercamiento y la coherencia entre las actividades y estrategias contempladas en las propuestas teórico-metodológicas de la Extensión Crítica. Si bien en el aula universitaria se pueden construir procesos activos, el trabajo en territorio y los conceptos presentados aportaron un gran plus a la tarea educativa en general y a la formación de una ciudadanía crítica y socialmente comprometida con la realidad regional, en particular. Dada la riqueza de esta experiencia creemos que un aporte fundamental a las instancias de formación profesional y a la producción de conocimientos es la inclusión de las prácticas extensionistas en la currícula de las carreras universitarias. Estas trayectorias de formación no pueden estar al margen del reconocimiento oficial por parte de las Universidades y del Ministerio de Educación.

\section{Referencias bibliográficas}

Argentina, Ley de Educación Nacional 26.206 (2006). Recuperado de https://www.argentina.gob.ar/sites/default/files/ley-de-educ-nac58 ac89392ea4c.pdf 
Ley de Enseñanza de Portugués 26.468 (2008). Recuperado de http://servicios.infoleg.gob.ar/infolegInternet/verNorma.do?id=149451 Arnoux, E. (2017). Integraciones regionales sudamericanas: Mercosur y Alianza del Pacífico. Politicas del lenguaje y discursos políticos. Publicación extraordinaria, Instituto de Investigaciones Lingüísticas y Literarias de la Patagonia (ILLPAT), Facultad de Humanidades y Ciencias Sociales, Universidad Nacional de la Patagonia San Juan Bosco.

Brasil, Ley de Enseñanza de español 11.161 (2005). Recuperado de http://www.planalto.gov.br/ccivil_03/_Ato2004-2006/2005/Lei/L11161.htm Bronckart, J. P., \& Dolz-Mestre, J. (2007). La noción de competencia: su pertinencia para el estudio del aprendizaje de la acciones verbales. Desarrollo del lenguaje y didáctica de las lenguas.

Canteros, A. (2011). Relações bilaterais, políticas culturais e ensino de PLE na argentina: da cooperação à integração e ao diálogo intercultural. Santa Fe: Ponencia Consiple.

CDC. (2009). Para la renovación de la enseñanza y la curricularización de la extensión y las actividades en el medio. Resolución №5, Consejo Directivo Central de la Universidad de la República. 27 de octubre de 2009. Recuperado de http://www.expe.edu.uy/expe/resoluci.nsf/e3365ff03c2a3d6103256dcc003b9031/f 200247a7f556d23032576550069e060?OpenDocument

Castro-Gómez, S. (2007). Decolonizar la universidad. La hybris del punto cero y el diálogo de saberes (pp. 79-91). En Castro-Gómez, Santiago \& Grosfoguel, Ramon (coords.). El giro decolonial: reflexiones para una diversidad epistémica más allá del capitalismo global . Bogotá: Siglo del Hombre, Universidad Central, Instituto de Estudios Sociales Contemporáneos, Pontificia Universidad Javeriana, Instituto Pensar.

http://www.unsa.edu.ar/histocat/hamoderna/grosfoguelcastrogomez.pdf

De Paula, J. A. (2013). A extensão universitária: história, conceitos e propostas. Interfaces - Revista de Extensão, 1(1), 05-23.

Del Valle, J. (2007). Glotopolítica, ideología y discurso: categorías para el estudio del estatus simbólico del español (pp. 13-30). Vervuert.

Fals Borda, O. (1986). Conocimiento y poder popular lecciones con campesinos de Nicaragua, México y Colombia (No. 630.715 F35).

Freire, P. (1987). Pedagogía del oprimido. Paz y Tierra. Río de Janeiro, Brasil.

Ghiso, A. (1998). De la práctica singular al diálogo con lo plural. Aproximaciones a otros tránsitos y sentidos de la sistematización en épocas de globalización. En Red de Bibliotecas virtuales de CLACSO http://www.cepalforjaorg/sistem/bvirtual/?p=661 
Jara, O. (2013). A sistematização de experiências, prática e teoria para outros mundos possiveis. Ed. CONTAG, Confederação Nacional dos Trabalhos da Agricultura. Brasília, Brasil.

UDELAR (Universidad de la República) (2010). Hacia la Reforma universitaria. La extensión en la renovación. Uruguay.

Lander, E. (2000). La colonialidad del saber: eurocentrismo y las ciencias sociales. Perspectivas latinoamericanas. CLACSO.

Quijano, A. (2013). El moderno Estado-nación en América Latina: cuestiones pendientes. Lima, Perú: Yuyaykusun.

Quijano, A. (2000). Colonialidad del poder y clasificación social. Journal of WorldSystem Research. (2): 342-386.

Quijano, A. (1992). Colonialidad y modernidad/racionalidad. Perú Indígena. 13, 29. Lima, Perú.

Rubio Scola, V. (2019a). Politicas Lingüísticas e integración regional: la enseñanza del portugués en Argentina. En Arnoux, E. Bein, R. (Org.) Peronismo y glotopolítica: intervenciones en el sistema educativo y las academias. Buenos Aires: Biblos, 2019.

Rubio Scola, V. (2019b). Tensiones ideológicas en materiales didácticos de portugués en Argentina - globalización, lusofonía, Mercosur. Línguas e instrumentos lingüísticos. $\mathrm{N}^{\circ}$ 43, Campinas: Unicamp, 2019, pp. 237-258. Recuperado de http://www.revistalinguas.com/edicao43/capa\%20edicao43.pdf

Sousa Santos, B. (2009). Una epistemología del sur. Siglo XXI. Clacso. México.

Walsh, C. (2008). Interculturalidad, plurinacionalidad y decolonialidad: las insurgencias politico-epistémicas de refundar el estado. Tabula Rasa. No 9. Bogotá, Colombia.

Zúñiga López, R. E., Zúñiga Preciado, M.T. (2013). Metodología para la Sistematización Participativa de Experiencias Sociales. Una propuesta desde la Educación Popular. IMDEC. Recuperado de http://www.congresoed.org/wpcontent/uploads/2014/10/Manual_Sistematizacion_2013_IMDEC.pdf 